Supporting Information for

\title{
Conflicting roles of anion doping on the electrochemical performance of Li-ion battery cathode materials
}

Fantai Kong, ${ }^{\dagger}$ Chaoping Liang, ${ }^{\dagger}$ Roberto C. Longo, ${ }^{\dagger}$ Dong-Hee Yeon, ${ }^{\ddagger}$ Yongping Zheng, ${ }^{\dagger}$ Jin-Hwan Park ${ }^{\ddagger}$ Seok-Gwang Doo*, ${ }^{*}$ and Kyeongjae Cho*,

${ }^{\dagger}$ Materials Science \& Engineering Dept., The University of Texas at Dallas, Richardson, TX 75080, United States

Energy Lab., Samsung Advanced Institute of Technology, Samsung Electronics, Yongin 446-712, Republic of Korea

KEYWORDS: $\mathrm{LiNiO}_{2}$, Sulfur, Fluorine, Chlorine, cathode materials

*Corresponding author. E-mail: kjcho@utdallas.edu (KJ. Cho); sgdoo@samsung.com (S.-G. Doo) 

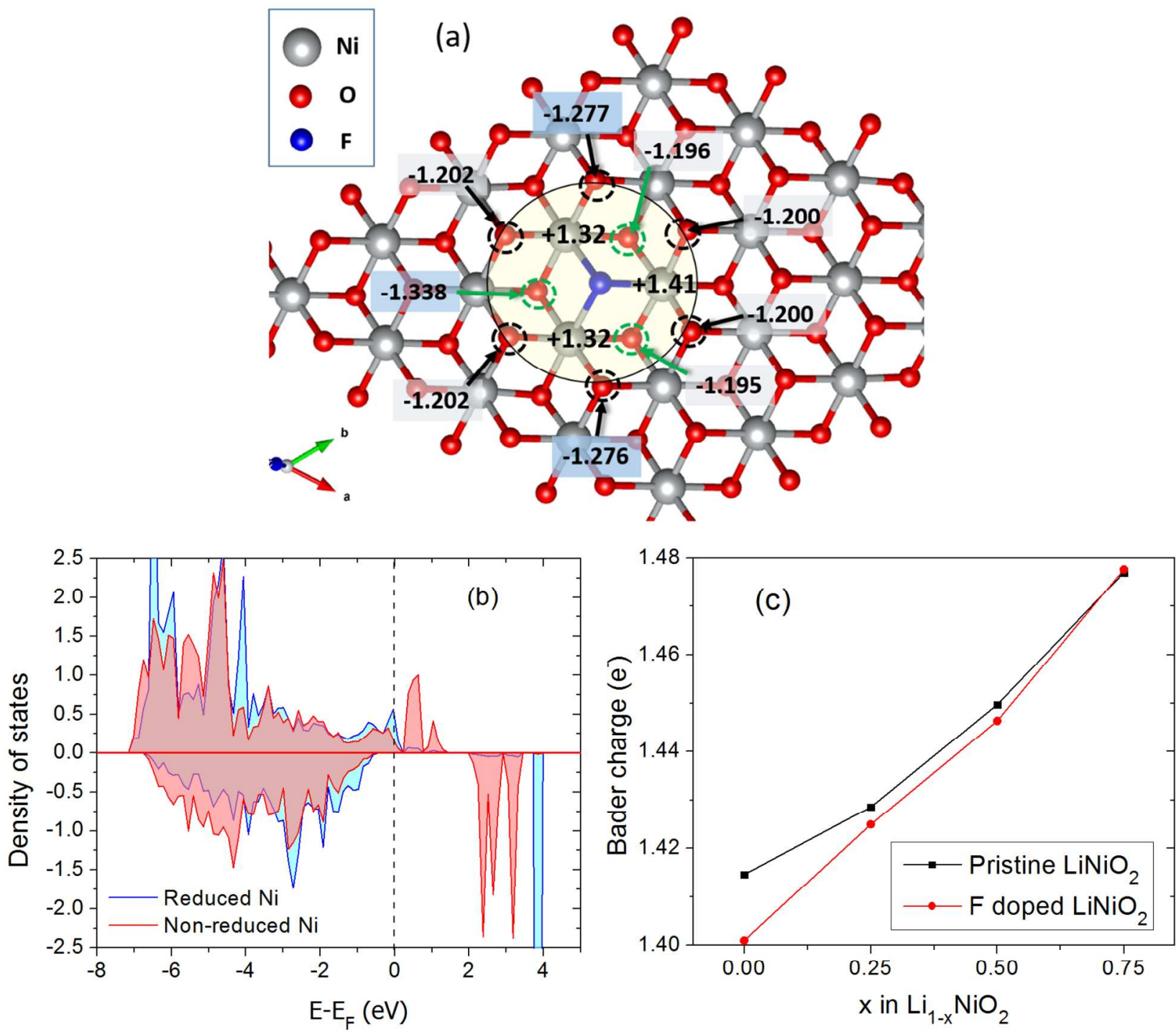

Figure S1. (a) Bader charge distribution of the ions neighboring with $\mathrm{F}$ in $\mathrm{F}$ doped $\mathrm{LiNiO}_{2}$. (b) Density of states of reduced and non-reduced $\mathrm{Ni}$ ions in $\mathrm{F}$ doped $\mathrm{LiNiO}_{2}$. (c) Average Bader charge of $\mathrm{Ni}$ ions as a function of delithiation concentration in pristine and $\mathrm{F}$ doped $\mathrm{LiNiO}_{2}$.

Figure $\mathrm{S} 1 \mathrm{a}$ shows that two $\mathrm{F}$ neighboring $\mathrm{Ni}$ ions are reduced (the average charge of $\mathrm{Ni}$ is $+1.41 \mathrm{e}^{-}$). At the same time, $\mathrm{O}$ ions bonding with the reduced $\mathrm{Ni}$ ions also show more electron transfer (the average charge of $\mathrm{O}$ is $-1.20 \mathrm{e}^{-}$). This result indicates the formation of small electron polaron in the $\mathrm{Ni}-\mathrm{O}$ bonds neighboring to the $\mathrm{F}$ dopant. Additionally, DOS of Ni ions plotted in Figure S1b supports the reduction of the $\mathrm{Ni}$ ions, resembling an oxidation state of $\mathrm{Ni}^{2.5+}$. The charge state in Figure S1a implies that the $\mathrm{F}$ dopant will also strengthen attractive $\mathrm{Li}-\mathrm{O}$ bonds while weaken repulsive $\mathrm{Ni}$-Li bonds, thus increasing the battery redox potential. 
Figure S1c shows the average Bader charge of $\mathrm{Ni}$ ions during delithiation. It indicates that the $\mathrm{F}$ effect of reducing $\mathrm{Ni}$ ions decreases during delithiation. Therefore, the increased voltage effect aforementioned will be weakened at the end of charge. Actually, at the end of delithiation, average oxidation state of $\mathrm{Ni}$ ions seems to be larger, which might be partially responsible for the slightly lower redox potential at this last stage of charging (see Figure 2).
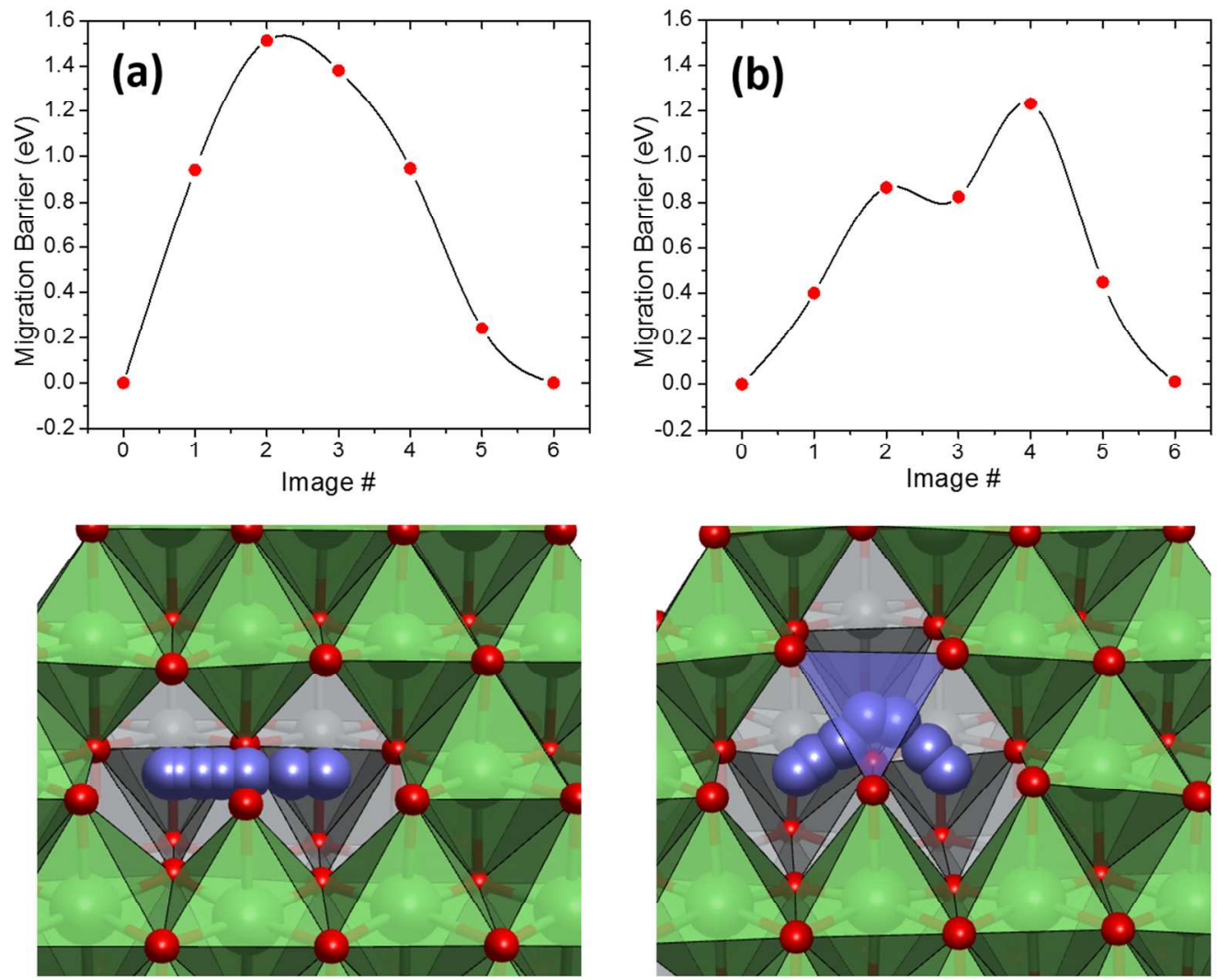

Figure S2. (a) Ni migration in Li layer, (b) Ni migration in Li layer with a neighboring Li vacancy. Purple sphere denotes the migrating $\mathrm{Ni}$ atom.

For Ni migration in the Li layer, two migration paths have been considered: direct migration and divacancy migration. Figure S2 shows that the kinetic barriers are as high as 1.3-1.5 eV, indicating that $\mathrm{Ni}$ would remain immobile at the $\mathrm{Li}$ site after the $\mathrm{Li} / \mathrm{Ni}$ anti-site defect is created. 


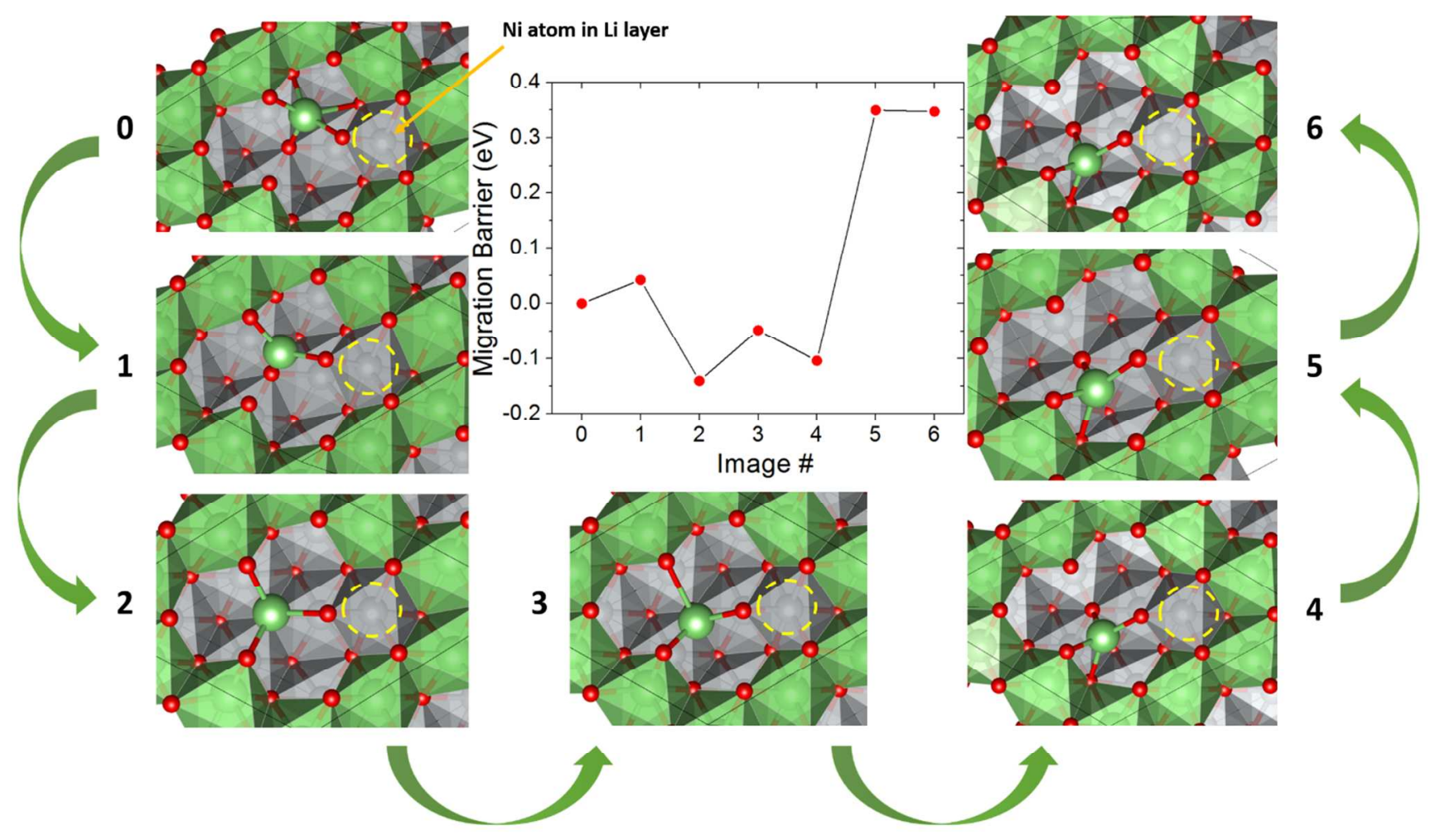

Figure S3. Li migration with a neighboring Ni atom in the Li layer.

Figure S3 shows the NEB results of the diffusion of a $\mathrm{Li}$ ion neighboring to a $\mathrm{Ni}$ atom in the $\mathrm{Li}$ layer. The Ni atom at the Li layer, with a higher oxidation state of $2+$, shows a strong repulsive force over the migrating $\mathrm{Li}$ ion. The $\mathrm{Li}$ ion is not stable at the octahedral $\mathrm{O}_{\mathrm{h}}$ site in both the initial and final states of the diffusion pathway, it occupies the intermediate $T_{d}$ site due to the larger distance with the $\mathrm{Ni}$ atom. The final migration barrier goes up to $0.45 \mathrm{eV}$, which is 0.12 eV higher than that in $\mathrm{LiNiO}_{2}$ without $\mathrm{Li} / \mathrm{Ni}$ anti-site defects. This result let us conclude that $\mathrm{Li}$ ions could still migrate around the anti-site defect, but with a higher activation barrier. 


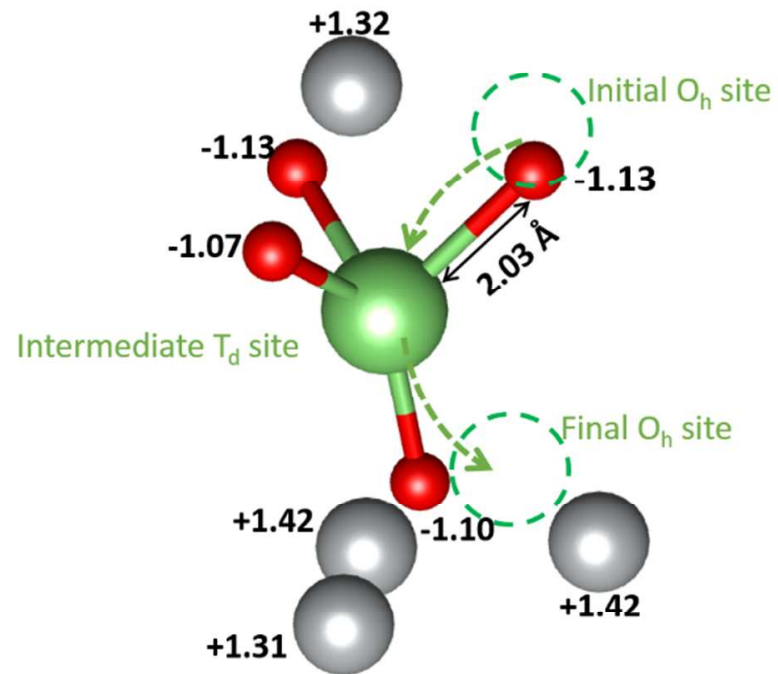

Pristine $\mathrm{LiNiO}_{2}$

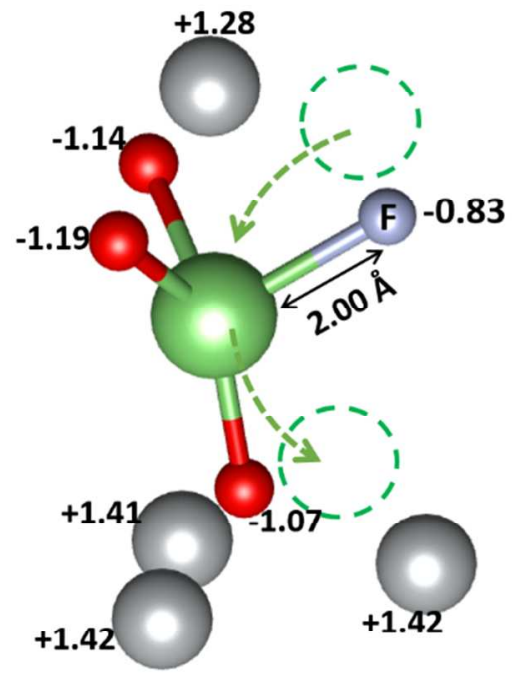

F-doped $\mathrm{LiNiO}_{2}$

\section{Saddle point structure}

Figure S4. Local configuration of the saddle point structure during $\mathrm{Li}$ migration in pristine $\mathrm{LiNiO}_{2}$ and F-doped $\mathrm{LiNiO}_{2}$, and the corresponding bader charge $\left(\mathrm{e}^{-}\right)$distributions.

Figure S4 shows the saddle point structure of pristine and F-doped $\mathrm{LiNiO}_{2}$. The bader charge distribution of the saddle point neighboring cations and anions is also shown. By comparing both situations, we can infer that the charge distribution of $\mathrm{Ni}$ and $\mathrm{O}$ in both cases is relatively similar. However, the bader charge of $F$ is $0.3 \mathrm{e}^{-}$larger than that of $\mathrm{O}$. This fact, along with similar $\mathrm{Li}-\mathrm{O}$ and $\mathrm{Li}-\mathrm{F}$ bond lengths, indicates a weaker resistance for Li migration at the saddle point, which might result in a lower migration barrier. Note that $\mathrm{Li}-\mathrm{O}$ bond length in $T_{d}$ site $(2.03 \AA)$ is much shorter than at the $\mathrm{O}_{h}$ site $(2.12 \AA)$. 


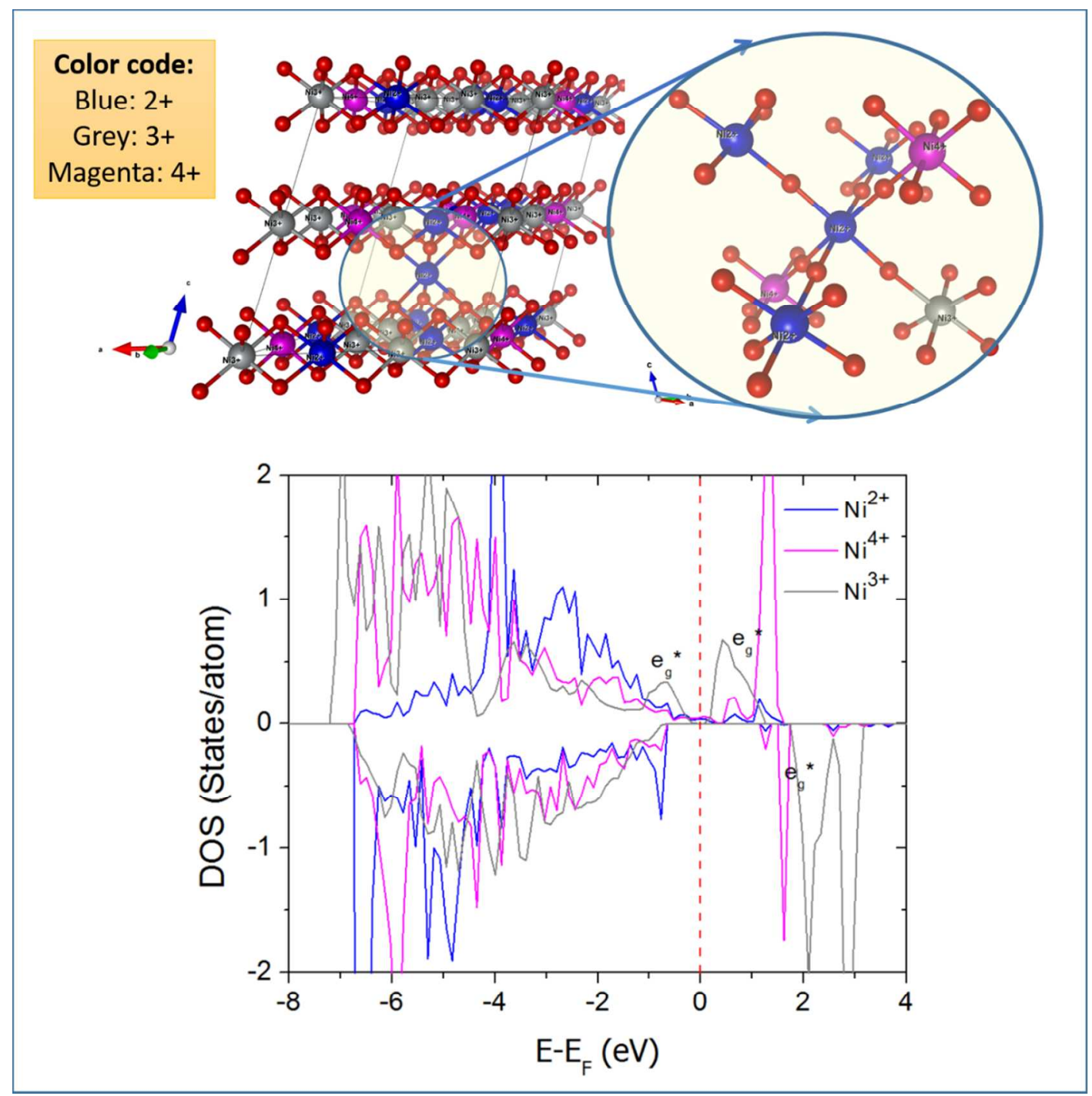

Figure S5. Charge distribution of $\mathrm{Ni}$ ions neighboring to a $\mathrm{Li} / \mathrm{Ni}$ anti-site defect pair, and DOS for $\mathrm{Ni}$ ions with different oxidation states.

Figure $\mathrm{S} 5$ shows that when two $\mathrm{Ni}^{3+}$ ions are reduced to $\mathrm{Ni}^{2+}$, the charge compensation is through neighboring $\mathrm{Ni}^{3+}$ ions, which are oxidized to $\mathrm{Ni}^{4+}$. The oxidation states of $\mathrm{Ni}$ ions are determined by combining both DOS and Bader charge analysis. 